\title{
Compatible families of elliptic type
}

\author{
by
}

\author{
DAvid E. Rohrlich (Boston, MA)
}

In axiomatizing their study of Frobenius distributions [5], Lang and Trotter introduce the notion of an adelic Galois representation of elliptic type, and they ask in passing whether every such representation arises from an elliptic curve (see pp. 5 and 19 of [5]). Formulated in the language of $\ell$-adic representations [7, their question is as follows. Put $G=\operatorname{Gal}(\overline{\mathbb{Q}} / \mathbb{Q})$, let $p$ denote a prime, and write $\sigma_{p}$ for any Frobenius element at a prime ideal of $\overline{\mathbb{Q}}$ over $p$. Let $\left\{\rho_{\ell}\right\}$ be a two-dimensional strictly compatible family of integral $\ell$-adic representations of $G$ with exceptional set $S$, and for $p \notin S$ put $a(p)=\operatorname{tr} \rho_{\ell}\left(\sigma_{p}\right)$ with any $\ell \neq p$. Also, let $\omega_{\ell}: G \rightarrow \mathbb{Z}_{\ell}^{\times}$denote the $\ell$-adic cyclotomic character. Although $\rho_{\ell}$ is a priori a map into $\mathrm{GL}\left(2, \mathbb{Q}_{\ell}\right)$, after a conjugation in $\mathrm{GL}\left(2, \mathbb{Q}_{\ell}\right)$ we may regard it as a map $G \rightarrow \mathrm{GL}\left(2, \mathbb{Z}_{\ell}\right)$.

Question of LANG AND TrotTer. Suppose that $\left\{\rho_{\ell}\right\}$ satisfies three conditions:

LT1. For $p \notin S \cup\{\ell\}$, $\operatorname{det} \rho_{\ell}\left(\sigma_{p}\right)=p$. In other words, $\operatorname{det} \rho_{\ell}=\omega_{\ell}$.

LT2. For $p \notin S,|a(p)|<2 \sqrt{p}$.

LT3. The image of $\rho_{\ell}$ is an open subgroup of $\mathrm{GL}\left(2, \mathbb{Z}_{\ell}\right)$ for every $\ell$ and is equal to $\mathrm{GL}\left(2, \mathbb{Z}_{\ell}\right)$ for all but finitely many $\ell$.

Does it follow that $\left\{\rho_{\ell}\right\}$ is isomorphic to the strictly compatible family $\left\{\rho_{E, \ell}\right\}$ afforded by the $\ell$-adic Tate modules $T_{\ell}(E)$ of some elliptic curve $E$ over $\mathbb{Q}$ ?

Here two strictly compatible families $\left\{\rho_{\ell}\right\}$ and $\left\{\rho_{\ell}^{\prime}\right\}$ are understood to be isomorphic if for each $\ell$ the representations $\rho_{\ell}$ and $\rho_{\ell}^{\prime}$ are isomorphic over $\mathbb{Q}_{\ell}$.

If we further stipulate that $E$ should not have complex multiplication then the question is simply whether certain necessary conditions for $\left\{\rho_{\ell}\right\} \cong$ $\left\{\rho_{E, \ell}\right\}$ are also sufficient. Indeed, LT1 and LT2 hold for any elliptic curve over $\mathbb{Q}$, the former being a consequence of the Galois-equivariance of the Weil pairing and the latter an instance of Hasse's Riemann hypothesis for

2010 Mathematics Subject Classification: Primary 11F80, 11 G05.

Key words and phrases: elliptic curve, Galois representation, compatible family. 
elliptic function fields (a strict inequality here because $\sqrt{p} \notin \mathbb{Q}$ ). As for LT3, if $E$ does not have complex multiplication then the fact that $\rho_{E, \ell}$ is open for all $\ell$ and surjective for all but finitely many $\ell$ is Serre's theorem $[8]$.

Elliptic curves with complex multiplication do not fall within the purview of the Lang-Trotter question, but we can include them simply by omitting LT3. The question is then whether families of the form $\left\{\rho_{E, \ell}\right\}$ are characterized by LT1 and LT2 alone. We shall see that an affirmative answer follows from the Fontaine-Mazur conjecture [1] combined with a "catalyst."

The version of the Fontaine-Mazur conjecture that is relevant here is the two-dimensional case stated on pp. 190-191 of [1]. As usual, we call a two-dimensional representation $\rho$ of $G$ even or odd according as $\operatorname{det} \rho$ is trivial or nontrivial on the conjugacy class of complex conjugation. And we shall refer to $\rho$ as an Artin representation if it factors through $\operatorname{Gal}(K / \mathbb{Q})$ for some finite Galois extension $K$ of $\mathbb{Q}$, even if the field of scalars of $\rho$ is not necessarily $\mathbb{C}$.

FM. Fix a prime $p$ and suppose that $\rho: G \rightarrow \mathrm{GL}\left(2, \overline{\mathbb{Q}}_{p}\right)$ is an irreducible representation which is potentially semistable at $p$ and unramified at all but finitely many primes of $\mathbb{Q}$. Assume also that $\rho$ does not have the form $\rho \cong \lambda \otimes \omega_{p}^{n}$, where $n \in \mathbb{Z}$ and $\lambda$ is an even Artin representation of $G$. Then there exists a primitive cusp form $f$ such that the associated semisimple representation $\rho_{f, p}$ is isomorphic to $\rho$.

Here "cusp form" means "cusp form of type $(N, k, \chi)$ for some positive integers $N$ and $k$ and Dirichlet character $\chi$ modulo $N$." Furthermore, if we write the Fourier expansion of $f$ as $f(z)=\sum_{n \geq 1} a(n) e^{2 \pi i n z}$ then we have implicitly fixed an embedding into $\overline{\mathbb{Q}}_{p}$ of the number field generated by the coefficients $a(n)$ and the values of $\chi$. It is then meaningful to specify that for primes $q \nmid N p$ we have $\operatorname{tr} \rho_{f, p}\left(\sigma_{q}\right)=a(q)$ and $\operatorname{det} \rho_{f, p}\left(\sigma_{q}\right)=\chi(q) q^{k-1}$. Since $\rho_{f, p}$ is semisimple it is determined up to isomorphism by these properties.

The catalyst that we need does not appear to have been enunciated in the literature, but it is implicit in the dual use of the word ordinary in contemporary arithmetic geometry. For the sake of clarity, let us call a prime $p \notin S$ classically ordinary (relative to $\left\{\rho_{\ell}\right\}$ ) if $p \nmid a(p)$, and ordinary (again, relative to $\left.\left\{\rho_{\ell}\right\}\right)$ if $p$ satisfies the definition on pp. 97-98 of Greenberg [2]. These notions are complementary in the sense that the former is a condition on $\rho_{\ell}$ for $\ell \neq p$ and the latter a condition on $\rho_{p}$. Nonetheless, we consider the following hypothesis:

ORD. Classically ordinary primes are ordinary.

While ORD lacks the legitimacy conferred by an eponym, it seems indispensable in the following application of $\mathbf{F M}$ : 
Theorem 1. Assume FM and ORD. If $\left\{\rho_{\ell}\right\}$ satisfies LT1 and LT2 then there is an elliptic curve $E$ over $\mathbb{Q}$ such that $\left\{\rho_{\ell}\right\} \cong\left\{\rho_{E, \ell}\right\}$.

The proof of Theorem 1 quickly reduces to an elementary remark. Let $\Lambda$ be the set of primes $\ell$ such that $\rho_{\ell}$ is absolutely irreducible, and let $\Lambda_{\text {surj }}$ be the subset of $\Lambda$ consisting of those $\ell$ for which $\rho_{\ell}(G)=\operatorname{GL}\left(2, \mathbb{Z}_{\ell}\right)$.

Theorem 2. Suppose that $\left\{\rho_{\ell}\right\}$ satisfies LT1 and LT2. Then $\Lambda$ has density 1 , and if there exists a prime $\ell_{0}$ such that $\rho_{\ell_{0}}(G)$ is open in $\operatorname{GL}\left(2, \mathbb{Z}_{\ell_{0}}\right)$ then $\Lambda_{\text {surj }}$ has density 1.

Only the density of $\Lambda$ is needed in the proof of Theorem 1, but the contingent density of $\Lambda_{\text {surj }}$ can be viewed as a weak form of LT3, which in this weak form is therefore a consequence of LT1 and LT2. Thus even without assuming FM or ORD there is reason to pose the Lang-Trotter question with LT3 omitted.

The reader may wonder why no use is made in this note of the extraordinary recent work of Kisin [3] establishing FM under certain hypotheses. The answer is that two of the hypotheses in [3] do not mesh well with the problem at hand. Indeed, one of these hypotheses - the potential semistability of $\rho$-is intrinsic to $\mathbf{F M}$ itself, and our appeal to ORD is merely a cheap way of evading the difficulty. The second problematic hypothesis is condition (4) of Kisin's main theorem ([3, p. 642]), which is a purely technical assumption and may well be eliminated in the not-too-distant future but which for the moment makes it difficult to apply [3] in any context where a Galois representation is expected to arise from an elliptic curve.

The preceding paragraph stems from a request for clarification by the referee, who also hinted that an explicit reminder regarding Greenberg's definition might be more helpful to the reader than a mere reference to [2. So fix a prime ideal of $\overline{\mathbb{Q}}$ over $p$ and let $G_{p} \subset G$ be the corresponding decomposition group, which we identify with $\operatorname{Gal}\left(\overline{\mathbb{Q}}_{p} / \mathbb{Q}_{p}\right)$. Also write $V_{p}$ for the space of $\rho_{p}$ and view $\rho_{p}$ as a representation of $\operatorname{Gal}\left(\overline{\mathbb{Q}}_{p} / \mathbb{Q}_{p}\right)$ via restriction to $G_{p}$. We say that $p$ is ordinary if there is a descending filtration of $V_{p}$ by $G_{p}$-stable $\mathbb{Q}_{p}$-subspaces $F^{i} V_{p}(i \in \mathbb{Z})$ such that $F^{i} V_{p}=\{0\}$ for $i$ sufficiently large, $F^{i} V_{p}=V_{p}$ for $i$ sufficiently small, and the restriction of $\rho_{p}$ to the inertia subgroup of $G_{p}$ acts on $F^{i} V_{p} / F^{i+1} V_{p}$ by the character $\omega_{p}^{i}$. Here $\omega_{p}^{i}$ is viewed as a character of the inertia subgroup by restriction.

1. Proof of Theorem 1 (granting Theorem 2). Given a prime $\ell$, let $\bar{\rho}_{\ell}$ denote the representation $G \rightarrow \mathrm{GL}\left(2, \mathbb{F}_{\ell}\right)$ obtained from $\rho_{\ell}$ by reduction modulo $\ell$. We begin the proof of Theorem 1 by fixing an odd prime $\ell_{0}$ and identifying $\bar{\rho}_{\ell_{0}}(G)$ with the Galois group over $\mathbb{Q}$ of the fixed field of the kernel of $\bar{\rho}_{\ell_{0}}$. Applying the Chebotarev density theorem to the one-element conjugacy class consisting of the identity $1 \in \bar{\rho}_{\ell_{0}}(G)$, we find that the set of 
primes $p \notin S \cup\left\{\ell_{0}\right\}$ such that $\bar{\rho}_{\ell_{0}}\left(\sigma_{p}\right)=1$ has positive density. Since we are granting that $\Lambda$ has density 1 , it follows that there is a prime $p \notin S \cup\left\{2,3, \ell_{0}\right\}$ such that $\rho_{p}$ is absolutely irreducible and $\bar{\rho}_{\ell_{0}}\left(\sigma_{p}\right)=1$. The latter condition implies that $a(p) \equiv 2 \bmod \ell_{0}$, and as $\ell_{0}$ is odd we deduce that $a(p) \neq 0$. Since $p \geq 5$ it follows from LT2 and the nonvanishing of $a(p)$ that $p \nmid a(p)$. In other words, $p$ is classically ordinary, hence ordinary by ORD, and therefore a theorem of Fontaine and Perrin-Riou [6] assures us that $\rho_{p}$ is semistable at $p$. Furthermore, $\rho_{p}$ is not the twist of an even Artin representation by some power of $\omega_{p}$, for then $\operatorname{det} \rho_{p}$ would be even, contrary to LT1. Thus FM is in force, and we can write $\rho_{p} \cong \rho_{f, p}$ for some primitive cusp form $f$. In fact, from LT1 we deduce that $f$ is of weight 2 with trivial character. And since $\operatorname{tr} \rho_{p}\left(\sigma_{q}\right)=a(q)$ for primes $q \notin S \cup\{p\}$, we obtain the further information that the Fourier coefficients of $f$ are rational integers, whence $\rho_{f, p} \cong \rho_{E, p}$ for some elliptic curve $E$ over $\mathbb{Q}$. Thus $\rho_{p} \cong \rho_{E, p}$. The proof of Theorem 1 is now completed by the following lemma.

Lemma. Let $\left\{\rho_{\ell}\right\}$ and $\left\{\rho_{\ell}^{\prime}\right\}$ be two strictly compatible families of $\ell$-adic representations of $G$, and suppose that $\rho_{p} \cong \rho_{p}^{\prime}$ for some prime $p$. Suppose in addition that $\rho_{\ell}^{\prime}$ is irreducible for every prime $\ell$. Then $\left\{\rho_{\ell}\right\} \cong\left\{\rho_{\ell}^{\prime}\right\}$.

Proof. The argument is standard, but we nonetheless recall it. Fix a prime $\ell$, and let $S$ and $S^{\prime}$ be the exceptional sets of the two families. For primes $q \notin S \cup S^{\prime} \cup\{\ell, p\}$ the strict compatibility of the two families gives $\operatorname{tr} \rho_{\ell}\left(\sigma_{q}\right)=\operatorname{tr} \rho_{p}\left(\sigma_{q}\right)$ and $\operatorname{tr} \rho_{\ell}^{\prime}\left(\sigma_{q}\right)=\operatorname{tr} \rho_{p}^{\prime}\left(\sigma_{q}\right)$, and since $\operatorname{tr} \rho_{p}=\operatorname{tr} \rho_{p}^{\prime}$ by hypothesis we deduce that $\operatorname{tr} \rho_{\ell}\left(\sigma_{q}\right)=\operatorname{tr} \rho_{\ell}^{\prime}\left(\sigma_{q}\right)$. It follows that $\operatorname{tr} \rho_{\ell}=\operatorname{tr} \rho_{\ell}^{\prime}$. Let $\rho_{\ell}^{\text {ss }}$ denote the semisimplification of $\rho_{\ell}$. By assumption, $\rho_{\ell}^{\prime}$ is irreducible and a fortiori semisimple, and since a semisimple representation in characteristic 0 is determined up to isomorphism by its trace, we obtain $\rho_{\ell}^{s s} \cong \rho_{\ell}^{\prime}$. This implies in particular that $\rho_{\ell}^{\text {ss }}$ is irreducible and so coincides up to isomorphism with $\rho_{\ell}$ itself. We conclude that $\rho_{\ell} \cong \rho_{\ell}^{\prime}$.

2. Proof of Theorem 2. As before, write $\bar{\rho}_{\ell}$ for the reduction of $\rho_{\ell}$ modulo $\ell$. In the following lemma, $\ell$ denotes a fixed prime.

Lemma 1. Consider prime numbers $p, p^{\prime} \notin S$, and put $d=a(p)^{2}-4 p$ and $d^{\prime}=a\left(p^{\prime}\right)^{2}-4 p^{\prime}$.

(a) If $\ell \nmid 2 p p^{\prime} d d^{\prime}$ and

$$
\left(\frac{d}{\ell}\right)=-\left(\frac{d^{\prime}}{\ell}\right)
$$

then $\bar{\rho}_{\ell}$ is absolutely irreducible.

(b) If in addition $\ell \nmid a(p) a\left(p^{\prime}\right)$ then the restriction of $\bar{\rho}_{\ell}$ to every subgroup of index 2 in $G$ is also absolutely irreducible. 
Proof. (a) Put $V=\mathbb{F}_{\ell}^{2}$, so that $V$ is the space of $\bar{\rho}_{\ell}$, and suppose on the contrary that there exists a one-dimensional $G$-stable subspace $W$ of $\overline{\mathbb{F}}_{\ell} \otimes_{\mathbb{F}_{\ell}} V$. We will obtain a contradiction by proving that $W$ is both defined over $\mathbb{F}_{\ell}$ (in other words, of the form $\overline{\mathbb{F}}_{\ell} \otimes_{\mathbb{F}_{\ell}} U$ for some subspace $U$ of $V$ ) and not defined over $\mathbb{F}_{\ell}$.

The characteristic polynomials of $\rho_{\ell}\left(\sigma_{p}\right)$ and $\rho_{\ell}\left(\sigma_{p^{\prime}}\right)$ are $x^{2}-a(p) x+p$ and $x^{2}-a\left(p^{\prime}\right) x+p^{\prime}$, whence the eigenvalues of $\bar{\rho}_{\ell}\left(\sigma_{p}\right)$ and $\bar{\rho}_{\ell}\left(\sigma_{p^{\prime}}\right)$ are the images in $\overline{\mathbb{F}}_{\ell}$ of the numbers

$$
\lambda_{ \pm}=\frac{a(p) \pm \sqrt{d}}{2}
$$

and

$$
\lambda_{ \pm}^{\prime}=\frac{a\left(p^{\prime}\right) \pm \sqrt{d^{\prime}}}{2}
$$

respectively. Applying the hypothesis to (1) and (2), we see that in one case the two eigenvalues are distinct elements of $\mathbb{F}_{\ell}$ while in the other case the eigenvalues are distinct elements of $\overline{\mathbb{F}}_{\ell}$ not belonging to $\mathbb{F}_{\ell}$. Now the fact that in both cases the eigenvalues are distinct implies that the corresponding eigenspaces are one-dimensional, and since $W$ is stable under $G$ it follows that $W$ is an eigenspace both of $\bar{\rho}_{\ell}\left(\sigma_{p}\right)$ and of $\bar{\rho}_{\ell}\left(\sigma_{p^{\prime}}\right)$. The rationality properties of the eigenvalues of $\bar{\rho}_{\ell}\left(\sigma_{p}\right)$ and $\bar{\rho}_{\ell}\left(\sigma_{p^{\prime}}\right)$ now imply the contradictory rationality properties of $W$ mentioned above, and we conclude that $\bar{\rho}_{\ell}$ is indeed absolutely irreducible.

(b) Suppose on the contrary that there is a subgroup $H$ of index 2 in $G$ and a one-dimensional subspace $W$ of $\overline{\mathbb{F}}_{\ell} \otimes_{\mathbb{F}_{\ell}} V$ which is stable under $H$. Then $\bar{\rho}_{\ell}(g)^{2}(W)=W$ for every $g \in G$. This holds in particular for $g=\sigma_{p}$ and $g=\sigma_{p^{\prime}}$, and for these two choices of $g$ the eigenvalues of $\bar{\rho}_{\ell}(g)^{2}$ can be read from (1) and (2): they are the images in $\overline{\mathbb{F}}_{\ell}$ of the numbers

$$
\left(\lambda_{ \pm}\right)^{2}=\frac{\left(a(p)^{2}-2 p\right) \pm a(p) \sqrt{d}}{2}
$$

and

$$
\left(\lambda_{ \pm}^{\prime}\right)^{2}=\frac{\left(a\left(p^{\prime}\right)^{2}-2 p^{\prime}\right) \pm a\left(p^{\prime}\right) \sqrt{d^{\prime}}}{2}
$$

respectively. Since $a(p)$ and $a\left(p^{\prime}\right)$ are by hypothesis nonzero modulo $\ell$, we see once again that in both cases the two eigenvalues are distinct. Hence the fact that the one-dimensional subspace $W$ is stable under $\bar{\rho}_{\ell}\left(\sigma_{p}\right)^{2}$ and $\bar{\rho}_{\ell}\left(\sigma_{p^{\prime}}\right)^{2}$ implies that $W$ is an eigenspace of both maps. But as before, one set of eigenvalues belongs to $\mathbb{F}_{\ell}$ and the other does not, so we have a contradiction.

In the next lemma we view $\mathbb{Q}^{\times} / \mathbb{Q}^{\times 2}$ as a vector space over $\mathbb{F}_{2}$. Given a prime $p \notin S$, we have $a(p)^{2}-4 p<0$ by LT2 and hence in particular 
$a(p)^{2}-4 p \neq 0$, so if we set $d=a(p)^{2}-4 p$ then we can consider the coset $d \mathbb{Q}^{\times 2}$ in $\mathbb{Q}^{\times} / \mathbb{Q}^{\times 2}$.

Lemma 2. Let $\mathcal{P}$ be a set of primes which contains $S$ and has density 0. There is a sequence $\left\{p_{i}\right\}_{i=1}^{\infty}$ of primes $p_{i} \notin \mathcal{P}$ such that the cosets of the numbers $d_{i}=a\left(p_{i}\right)^{2}-4 p_{i}$ are linearly independent in $\mathbb{Q}^{\times} / \mathbb{Q}^{\times 2}$.

Proof. We shall construct the sequence $\left\{p_{i}\right\}$ inductively. To start the induction, choose any prime $p_{1} \notin \mathcal{P}$. As just noted, the quantity $d_{1}=$ $a\left(p_{1}\right)^{2}-4 p_{1}$ is negative and hence not in $\mathbb{Q}^{\times 2}$. Thus the vector $d_{1} \mathbb{Q}^{\times 2}$ is nonzero.

Now suppose that for some $n \geq 1$ we have chosen primes $p_{1}, \ldots, p_{n} \notin \mathcal{P}$ such that the cosets of $d_{1}, \ldots, d_{n}$ in $\mathbb{Q}^{\times} / \mathbb{Q}^{\times 2}$ are linearly independent. Then the Chebotarev density theorem ensures that the set of primes $p \nmid 2 d_{1} \cdots d_{n}$ such that

$$
\left(\frac{d_{1}}{p}\right)=\cdots=\left(\frac{d_{n}}{p}\right)=-1
$$

has density $2^{-n}$ and in particular positive density. Hence we can choose a prime $p_{n+1} \notin \mathcal{P}$ such that (3) holds with $p=p_{n+1}$. Put $d_{n+1}=a\left(p_{n+1}\right)^{2}-$ $4 p_{n+1}$. We must show that the vector $d_{n+1} \mathbb{Q}^{\times 2}$ is not in the span of the vectors $d_{i} \mathbb{Q}^{\times 2}(1 \leq i \leq n)$.

Suppose on the contrary that for some choice of exponents $\epsilon_{i} \in\{0,1\}$ $(1 \leq i \leq n)$ and some choice of $v \in \mathbb{Q}^{\times}$we have

$$
d_{n+1}=d_{1}^{\epsilon_{1}} \cdots d_{n}^{\epsilon_{n}} \cdot v^{2} .
$$

Then the quantity $\epsilon=\epsilon_{1}+\cdots+\epsilon_{n}$ is odd, because $d_{1}, \ldots, d_{n}<0$ and also $d_{n+1}<0$ while $v^{2}>0$. Thus on setting

$$
d=d_{1}^{\epsilon_{1}} \cdots d_{n}^{\epsilon_{n}}
$$

we have

$$
\left(\frac{d}{p_{n+1}}\right)=(-1)^{\epsilon}=-1,
$$

because by construction, (3) holds with $p=p_{n+1}$. It follows that $p_{n+1}$ remains prime in $\mathbb{Q}(\sqrt{d})$ and hence is not a norm from $\mathbb{Q}(\sqrt{d})$. This is a contradiction, for we can rewrite (40 in the form $p_{n+1}=\left(u^{2}-d v^{2}\right) / 4$ with $u=a\left(p_{n+1}\right)$.

Let $\Pi$ be the set of primes $p \notin S$ such that $a(p)=0$. If $\Pi$ has density 0 then let $\mathcal{L}$ denote the set of primes $\ell$ such that $\bar{\rho}_{\ell} \mid H$ is absolutely irreducible for every subgroup $H$ of index 2 in $G$. If the upper density of $\Pi$ is strictly positive then we define $\mathcal{L}$ by requiring only that $\bar{\rho}_{\ell}$ itself be absolutely irreducible. In both cases $\mathcal{L} \subset \Lambda$, so the first assertion of Theorem 2 is a consequence of the next lemma:

Lemma 3. $\mathcal{L}$ has density 1. 
Proof. We apply Lemma 2 with

$$
\mathcal{P}= \begin{cases}\Pi \cup S & \text { if } \Pi \text { has density } 0, \\ S & \text { otherwise }\end{cases}
$$

obtaining sequences $\left\{p_{i}\right\}$ and $\left\{d_{i}\right\}$ with $p_{i} \notin \mathcal{P}$ and $d_{i}=a\left(p_{i}\right)^{2}-4 p_{i}$ such that for every $n \geq 1$ the Galois group of $\mathbb{Q}\left(\sqrt{d_{1}}, \ldots, \sqrt{d_{n}}\right)$ over $\mathbb{Q}$ is $(\mathbb{Z} / 2 \mathbb{Z})^{n}$. Let

$$
c_{n}= \begin{cases}2 \prod_{i=1}^{n} p_{i} d_{i} a\left(p_{i}\right) & \text { if } \Pi \text { has density } 0, \\ 2 \prod_{i=1}^{n} p_{i} d_{i} & \text { otherwise. }\end{cases}
$$

Then $c_{n} \neq 0$. We put $\mathcal{M}_{n}=\mathcal{M}_{n}^{+} \cup \mathcal{M}_{n}^{-}$, where $\mathcal{M}_{n}^{ \pm}$is the set of primes $\ell \nmid c_{n}$ such that

$$
\left(\frac{d_{1}}{\ell}\right)=\cdots=\left(\frac{d_{n}}{\ell}\right)= \pm 1
$$

By the Chebotarev density theorem, $\mathcal{M}_{n}^{+}$and $\mathcal{M}_{n}^{-}$each have density $2^{-n}$, whence $\mathcal{M}_{n}$ has density $2^{1-n}$. It follows that the complement of $\mathcal{M}_{n}$ in the set of all primes not dividing $c_{n}$ has density $1-2^{1-n}$. Denote this complement $\mathcal{L}_{n}$. To prove the lemma it suffices to see that $\mathcal{L}_{n} \subset \mathcal{L}$, for then $\mathcal{L}$ has lower density $\geq 1-2^{1-n}$ with $n$ arbitrarily large. So suppose that $\ell \in \mathcal{L}_{n}$. Then $\ell \notin \mathcal{M}_{n}$, and consequently there are indices $i$ and $j(1 \leq i<j \leq n)$ such that

$$
\left(\frac{d_{i}}{\ell}\right)=-\left(\frac{d_{j}^{\prime}}{\ell}\right)
$$

Applying Lemma 1 with $p=p_{i}, p^{\prime}=p_{j}, d=d_{i}$, and $d^{\prime}=d_{j}^{\prime}$, we conclude that $\ell \in \mathcal{L}$.

It remains to prove the second assertion of Theorem 2, Let $\mathcal{L}^{*} \subset \mathcal{L}$ be the subset consisting of all $\ell \in \mathcal{L}$ such that $\bar{\rho}_{\ell}(G)$ either contains $\operatorname{SL}\left(2, \mathbb{F}_{\ell}\right)$ or is contained in the normalizer of a Cartan subgroup of $\mathrm{GL}\left(2, \mathbb{F}_{\ell}\right)$.

Lemma 4. $\mathcal{L}^{*}$ has density 1 . In fact, $\mathcal{L} \backslash \mathcal{L}^{*}$ is finite.

Proof. In view of Lemma 3 it suffices to prove the second statement. Now if $\ell \in \mathcal{L}$ then $\bar{\rho}_{\ell}$ is irreducible, and consequently $\bar{\rho}_{\ell}(G)$ is not contained in a Borel subgroup of $\operatorname{GL}\left(2, \mathbb{F}_{\ell}\right)$. If in addition $\ell \notin \mathcal{L}^{*}$ then $\bar{\rho}_{\ell}(G)$ neither contains $\operatorname{SL}\left(2, \mathbb{F}_{\ell}\right)$ nor is contained in the normalizer of a Cartan subgroup of $\operatorname{GL}\left(2, \mathbb{F}_{\ell}\right)$, whence the classification of subgroups of $\operatorname{GL}\left(2, \mathbb{F}_{\ell}\right)$ leaves only one possibility (cf. [8, p. 280, Prop. 15 and p. 282, 2.6]): For every $g \in G$ we have

$$
P\left(u_{\ell}(g)\right) \equiv 0 \bmod \ell
$$

where $P$ is the polynomial $P(u)=u(u-1)(u-2)(u-4)\left(u^{2}-3 u+1\right)$ and $u_{\ell}(g)=\left(\operatorname{tr} \rho_{\ell}(g)\right)^{2} / \operatorname{det} \rho_{\ell}(g)$. Suppose that $\mathcal{L} \backslash \mathcal{L}^{*}$ is infinite, so that (6) holds for infinitely many $\ell \in \mathcal{L}$. Then for $p \notin S$ and infinitely many $\ell \neq p$ 
we have

$$
P\left(u_{\ell}\left(\sigma_{p}\right)\right) \equiv 0 \bmod \ell .
$$

But $u_{\ell}\left(\sigma_{p}\right)=a(p)^{2} / p$, so $p^{6} P\left(u_{\ell}\left(\sigma_{p}\right)\right)$ is a rational integer, and since it is congruent to 0 for infinitely many $\ell$ it is equal to 0 . In other words, $Q(a(p))=0$, where $Q(x)=x^{2}\left(x^{2}-p\right)\left(x^{2}-2 p\right)\left(x^{2}-4 p\right)\left(x^{4}-3 p x^{2}+p^{2}\right)$. By inspection, the only rational root of $Q(x)=0$ is $x=0$, so $a(p)=0$ for all $p \notin S$. As we saw already in the proof of Theorem 1 1 this is impossible. (If we fix an odd prime $\ell_{0}$, then by the Chebotarev density theorem there are infinitely many $p \notin S \cup\left\{\ell_{0}\right\}$ such that $\bar{\rho}_{\ell_{0}}\left(\sigma_{p}\right)=1$, and for such $p$ we have $a(p) \equiv 2 \bmod \ell_{0}$.) We conclude that $\mathcal{L} \backslash \mathcal{L}^{*}$ is finite.

Lemma 5. Let $\ell$ be a prime $\geq 5$. If $\bar{\rho}_{\ell}(G)$ contains $\operatorname{SL}\left(2, \mathbb{F}_{\ell}\right)$ then $\rho_{\ell}(G)$ $=\mathrm{GL}\left(2, \mathbb{Z}_{\ell}\right)$.

Proof. If $\ell \geq 5$ and $X$ is a closed subgroup of $\operatorname{GL}\left(2, \mathbb{Z}_{\ell}\right)$ such that the reduction of $X$ modulo $\ell$ contains $\operatorname{SL}\left(2, \mathbb{F}_{\ell}\right)$ then $X$ contains $\operatorname{SL}\left(2, \mathbb{Z}_{\ell}\right)([7$, p. IV-23, Lemma 3], or see [4, p. 229]). In the case $X=\rho_{\ell}(G)$ our assumption LT1 then gives $\rho_{\ell}(G)=\mathrm{GL}\left(2, \mathbb{Z}_{\ell}\right)$.

The proof of Theorem 2 is completed by the next lemma.

Lemma 6. The following are equivalent:

(i) $\Lambda_{\text {surj }}$ has density 1.

(ii) There exists a prime $\ell_{0}$ such that $\rho_{\ell_{0}}(G)$ is open in $\operatorname{GL}\left(2, \mathbb{Z}_{\ell_{0}}\right)$.

(iii) $\Pi$ has density 0.

Proof. That (i) implies (ii) is trivial, and that (ii) implies (iii) follows from the Chebotarev density theorem (cf. [9, p. 150, Theorem 10], which is much stronger than what is needed here). Now suppose that $\Pi$ has density 0 . In view of Lemma 4 it will suffice to see that $\mathcal{L}^{*} \subset \Lambda_{\text {surj. }}$. In fact, by Lemma 5 we need only show that if $\ell \in \mathcal{L}^{*}$ then $\bar{\rho}_{\ell}(G)$ is not contained in the normalizer of a Cartan subgroup $C$ of $\mathrm{GL}\left(2, \mathbb{F}_{\ell}\right)$. If on the contrary such a containment does hold, then $\rho_{\ell}^{-1}(C)$ is a subgroup $H$ of index 2 in $G$ such that $\bar{\rho}_{\ell}(H)$ is abelian. It follows in particular that $\bar{\rho}_{\ell} \mid H$ is not absolutely irreducible. This contradicts the definition of $\mathcal{L}$ in the case where $\Pi$ has density 0.

Acknowledgments. This note began as a Boston University seminar talk given on October 16, 2006, and it is a pleasure to thank the seminar audience for indulging and enlightening me. I am particularly indebted to Avner Ash, Ben Howard, Jeehoon Park, Robert Pollack, Matthias Schütt, and Glenn Stevens, whose knowledgeable comments were collectively a much better talk than the one that was delivered. Further orientation was provided by Matt Emerton, Ralph Greenberg, and Kiran Kedlaya, whom it is also a pleasure to thank. Finally, I owe Robert Pollack a separate and special 
expression of gratitude for pointing out a serious error-pertaining precisely to condition (4) in [3] - in an earlier version of this note.

\section{References}

[1] J.-M. Fontaine and B. Mazur, Geometric Galois representations, in: Elliptic Curves, Modular Forms, \& Fermat's Last Theorem, J. Coates and S.-T. Yau (eds.), Int. Press, Cambridge, MA, 1997, 190-227.

[2] R. Greenberg, Iwasawa theory for p-adic representations, in: Algebraic Number Theory - in honor of K. Iwasawa, Adv. Stud. Pure Math. 17, Academic Press, 1989, 97-137.

[3] M. Kisin, The Fontaine-Mazur conjecture for $G L_{2}$, J. Amer. Math. Soc. 22 (2009), 641-690.

[4] S. Lang, Elliptic Functions, Grad. Texts in Math. 112, Springer, 1987.

[5] S. Lang and H. Trotter, Frobenius Distributions in GL $L_{2}$-Extensions, Lecture Notes in Math. 504, Springer, 1976.

[6] B. Perrin-Riou (with an appendix by L. Illusie), Représentations p-adiques ordinaires, in: Périodes p-adiques, Astérisque 223 (1994), 185-220.

[7] J.-P. Serre, Abelian $\ell$-Adic Representations and Elliptic Curves, W. A. Benjamin, 1968.

[8] - Propriétés galoisiennes des points d'ordre fini des courbes elliptiques, Invent. Math. 15 (1972), 259-331.

[9] —, Quelques applications du théorème de densité de Chebotarev, Publ. Math. IHES 54 (1981), 123-201.

David E. Rohrlich

Department of Mathematics and Statistics

Boston University

Boston, MA 02215, U.S.A.

E-mail: rohrlich@math.bu.edu

Received on 3.9.2007

and in revised form on 9.3.2009 